Delft University of Technology

\title{
Deep sub-wavelength metrology for advanced defect classification
}

van der Walle, P; Kramer, E.; van der Donck, J.C.J.; Mulckhuyse, W; Nijsten, L.; Bernal Arango, F. A.; de Jong, A.; van Zeijl, E.; Spruit, H. E.T.; van Den Berg, J. H.

DOI

10.1117/12.2272414

Publication date

2017

Document Version

Final published version

Published in

Optical Measurement Systems for Industrial Inspection X

\section{Citation (APA)}

van der Walle, P., Kramer, E., van der Donck, J. C. J., Mulckhuyse, W., Nijsten, L., Bernal Arango, F. A., de Jong, A., van Zeijl, E., Spruit, H. E. T., van Den Berg, J. H., Nanda, G., Van Langen-Suurling, A. K.,

Alkemade, P. F. A., Pereira, S. F., \& Maas, D. J. (2017). Deep sub-wavelength metrology for advanced defect classification. In P. Lehmann, W. Osten, \& A. Albertazzi Gonçalves (Eds.), Optical Measurement Systems for Industrial Inspection X [103294N] (Proceedings of SPIE; Vol. 10329). SPIE. https://doi.org/10.1117/12.2272414

Important note

To cite this publication, please use the final published version (if applicable).

Please check the document version above. 


\section{Deep sub-wavelength metrology for advanced defect classification}

P. van der Walle, E. Kramer, J. C. J. van der Donck, W. Mulckhuyse, L. Nijsten, et al.

P. van der Walle, E. Kramer, J. C. J. van der Donck, W. Mulckhuyse, L. Nijsten, F. A. Bernal Arango, A. de Jong, E. van Zeijl, H. E. T. Spruit, J. H. van den Berg, G. Nanda, A. K. van Langen-Suurling, P. F. A. Alkemade, S. F. Pereira, D. J. Maas, "Deep sub-wavelength metrology for advanced defect classification," Proc. SPIE 10329, Optical Measurement Systems for Industrial Inspection X, 103294N (26 June 2017); doi: 10.1117/12.2272414

SPIE. Event: SPIE Optical Metrology, 2017, Munich, Germany 


\title{
Deep sub-wavelength metrology for advanced defect classification
}

\author{
P. van der Walle ${ }^{a}$, E. Kramer, ${ }^{a, b}$ J.C.J. van der Donck ${ }^{a}$, W. Mulckhuyse ${ }^{a}$, L. Nijsten ${ }^{a}$, \\ F.A. Bernal Arango ${ }^{\mathrm{a}, \mathrm{b}}$, A. de Jong ${ }^{\mathrm{a}}$, E. van Zeijl ${ }^{\mathrm{a}}$, H.E.T. Spruit ${ }^{\mathrm{a}}$, J.H. van den Berg ${ }^{\mathrm{a}}$, G. Nanda ${ }^{\mathrm{b}}$, \\ A.K. van Langen-Suurling ${ }^{c}$, P.F.A. Alkemade ${ }^{c}$, S.F. Pereira ${ }^{b}$ and D.J. Maas ${ }^{* a}$ \\ TNO, 2626CK Delft, Netherlands; ${ }^{\mathrm{b}}$ Department of Imaging Physics, Faculty of Applied Sciences, \\ Delft University of Technology, Lorentzweg 1, 2628CJ Delft, Netherlands; ' Kavli Institute of \\ Nanoscience, Delft University of Technology, Lorentzweg 1, 2628CJ Delft, Netherlands
}

\begin{abstract}
Particle defects are important contributors to yield loss in semi-conductor manufacturing. Particles need to be detected and characterized in order to determine and eliminate their root cause. We have conceived a process flow for advanced defect classification (ADC) that distinguishes three consecutive steps; detection, review and classification. For defect detection, TNO has developed the Rapid Nano (RN3) particle scanner, which illuminates the sample from nine azimuth angles. The RN3 is capable of detecting $42 \mathrm{~nm}$ Latex Sphere Equivalent (LSE) particles on XXX-flat Silicon wafers. For each sample, the lower detection limit (LDL) can be verified by an analysis of the speckle signal, which originates from the surface roughness of the substrate. In detection-mode (RN3.1), the signal from all illumination angles is added. In review-mode (RN3.9), the signals from all nine arms are recorded individually and analyzed in order to retrieve additional information on the shape and size of deep sub-wavelength defects. This paper presents experimental and modelling results on the extraction of shape information from the RN3.9 multi-azimuth signal such as aspect ratio, skewness, and orientation of test defects.

Both modeling and experimental work confirm that the RN3.9 signal contains detailed defect shape information. After review by RN3.9, defects are coarsely classified, yielding a purified Defect-of-Interest (DoI) list for further analysis on slower metrology tools, such as SEM, AFM or HIM, that provide more detailed review data and further classification. Purifying the DoI list via optical metrology with RN3.9 will make inspection time on slower review tools more efficient.
\end{abstract}

Keywords: Particle contamination, defect detection, defect review, advanced defect classification, semiconductor, latex sphere equivalent, dark field microscopy, speckle.

\section{INTRODUCTION}

Particle defects are a significant contributor to yield loss in semiconductor manufacturing. To control particle defects, the presence, shape, size and composition of particles must be characterized. Advanced Defect Classification (ADC) is an established technique to install defectivity control ${ }^{1}$. With shrinking device dimensions, smaller and smaller particles become the root cause for critical defects. This strongly increases the challenge for defect detection and review tools, as both more and smaller particles need to be analyzed. This paper reports on the feasibility of fast ADC by optical metrology using RN3. The aim of ADC using RN3 is to purify the increasingly long list of defects-of-interest. Thus, the efficacy of more detailed defect review and classification by slower metrologies that have a higher resolution, such as e.g. SEM and AFM can be improved. Figure 1 shows our process flow for ADC: after defect detection and review, all defect signals are matched with physical properties of the defects, thus enabling classification. As a result of ADC by $\mathrm{RN} 3$, the industry will be better positioned to match the increasingly stringent requirements in defectivity control.

Under regular processing conditions, particles occur at ultra-low densities. Hence, fast optical inspection techniques are in use to scan large areas. For the cleanliness qualification of product modules and production processes, often blank witness wafers and/or reticles are used. TNO's RN3 particle scanner (see Figure 2) has been developed to detect particle defects down to $42 \mathrm{~nm}$ in diameter on flat un-patterned uniform substrates up to $150 \mathrm{~mm}$ in lateral size, such as e.g. 100 mm XXX-flat Si wafers or EUV mask blanks ${ }^{2}$. This extreme level of sensitivity is achieved by illuminating the sample from multiple angles, in a variant of dark-field microscopy. For each sample, the lower detection limit (LDL) is determined by the speckle signal, which originated from the surface roughness of the substrate.

Optical Measurement Systems for Industrial Inspection X, edited by Peter Lehmann, Wolfgang Osten, Armando Albertazzi Gonçalves Jr., Proc. of SPIE Vol. 10329, 103294N · @ 2017 SPIE CCC code: $0277-786 X / 17 / \$ 18 \cdot$ doi: $10.1117 / 12.2272414$ 

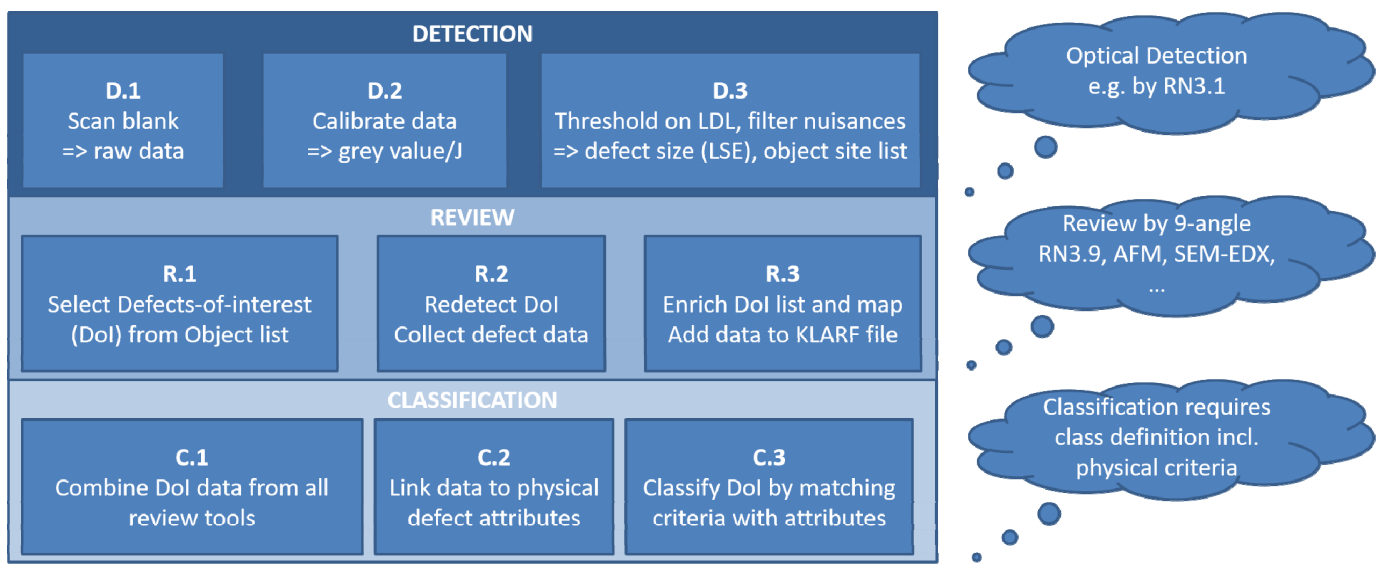

Figure 1 Process flow for the three stages in Detection, Review and Classification.

In detection-mode (RN3.1) the signal from all nine illumination angles is added. After a RN3.1 scan of the full substrate, the detected defects are ranked according to their importance in a defects of interests (DoI) list. In review-mode (RN3.9) the signals from all nine arms are processed individually and analyzed to retrieve additional information on the shape and size of defects, even for deep sub-wavelength-sized defects. This paper presents experimental and modelling results on the extraction of more information from the RN3.9 multi-azimuth signal such as the aspect ratio, skewness and orientation of the defect. After review by RN3.9, defects are coarsely classified, yielding a purified DoI list for further defect analysis, e.g. by $\mathrm{SEM}^{3}, \mathrm{AFM}^{4}$ or $\mathrm{HIM}^{5}$, which are all slower metrology tools that provide more detailed review data than RN3.9. To facilitate holistic connectivity between all defect review metrologies, a variant of the stealth fiducial marker system of Bouwens et al. ${ }^{6}$ has been developed.

\section{METHODS}

Figure 2 displays two pictures of TNO's Rapid Nano (RN3) particle scanner for blank substrates is based on dark field imaging ${ }^{2,7}$. The resulting signal is an image of the local scatter intensity for the uniformly illuminated field. The stage moves the substrate step-wise under the optical column, with sufficient overlap between the fields to stich all images into a defect map of the full blank substrate. The scatter intensity is converted into an estimated defect size, which is reported to the user in terms of a Latex Sphere Equivalent (LSE) diameter.

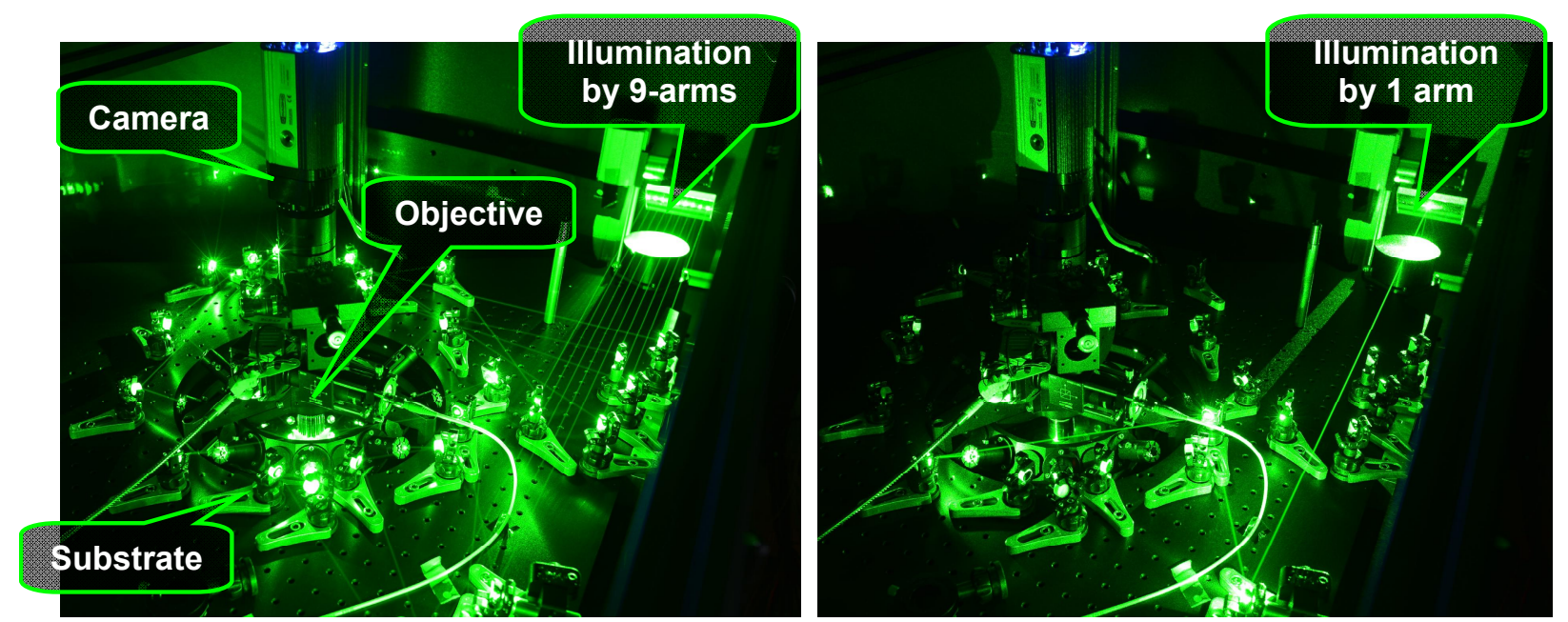

Figure 2 Pictures of TNO's RN3, illustrating the illumination through the nine arms that are arranged around the dark-field optical column. Left picture: annular illumination using all nine arms simultaneously (RN3.1 mode). Right picture: illumination using a single arm only (RN3.9 mode). 
A data acquisition mode for RN3 was developed to enable the extraction of the signals for each illumination angle separately, Furthermore, for ADC using optical data only, the relation of a defect's shape and composition to its optical scatter behavior into a dark field microscope objective needs to be established, at least for the most abundant and relevant defect classes. To this end, we studied the optical scattering from a set of programmed defects on a silicon wafer experimentally, and performed analytical as well as numerical finite element analysis modelling.

\subsection{Numerical model for the optical scattering from arbitrarily shaped defects}

A numerical model, based on finite element analysis (FEA) using $\mathrm{COMSOL}^{8}$, has been developed to calculate the optical scattering intensity from sub-wavelength-sized defects under realistic illumination and detection conditions for RN3.1 and RN3.9. The FEA model calculates the near-field around the defect and an open-source code is used to transfer this field to the far-field ${ }^{9,10}$. The validity of the numerical model was confirmed in a benchmark with the analytic BobbertVlieger ${ }^{11}$ model for the optical scattering from spherical particles on flat surfaces, see Figure 3. The model allows calculation of the scatter of sub-wavelength defects for a number of geometries, sizes, aspect ratios and materials.
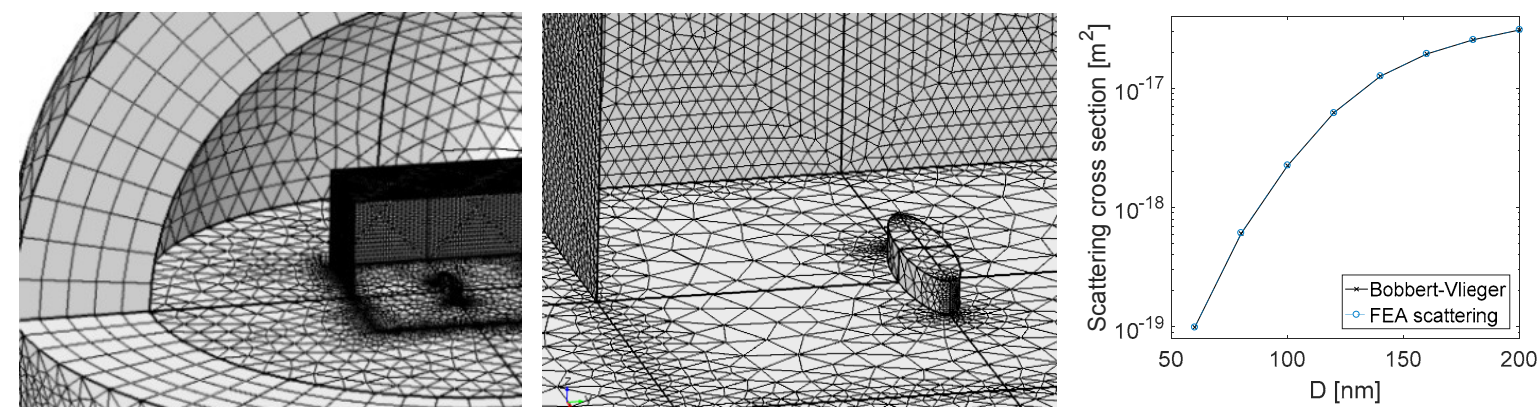

Figure 3 Left and center panel: mesh of numerical FEA model $\left(\mathrm{COMSOL}^{8}\right)$ as constructed to calculate the optical scattering from a sub-wavelength-sized elliptical cylinder into the pupil of the RN3. In the model, either annular or unidirectional illumination of a defect of arbitrary shape can be selected. Right panel: the prediction by the analytical Bobbert-Vlieger ${ }^{11}$ model for the scatter cross section for spherical Au particles with diameter D on a silicon substrate by is accurately reproduced by the numerical FEA model.

\subsection{Enhanced defect redetection efficacy using a stealth fiducial marker system}

In defect review, a high defect re-detection efficacy is of eminent importance. For this goal a fiducial marker system that is visible in all involved metrology sources has been developed. ${ }^{6}$ To be fully compliant with semiconductor manufacturing processes, a laser-scribing process has been optimized to write stealth fiducial markers on clean blank silicon wafers, see Figure 4. The marker system enables accurate and fast registration of the RN3 defect map on the stage coordinate system for each alternative metrology source ${ }^{6}$. The realized defect re-detection method has sufficient accuracy to facilitate defect review on blanks with RN3, AFM and SEM, thus providing an essential ingredient for holistic and hybrid metrology. To allow for accurate detection and localization on all inspection platforms stealth markers are required, i.e. the markers have a low scattering cross-section in dark-field microscopy, but are clearly visible in bright-field microscopy and SEM.
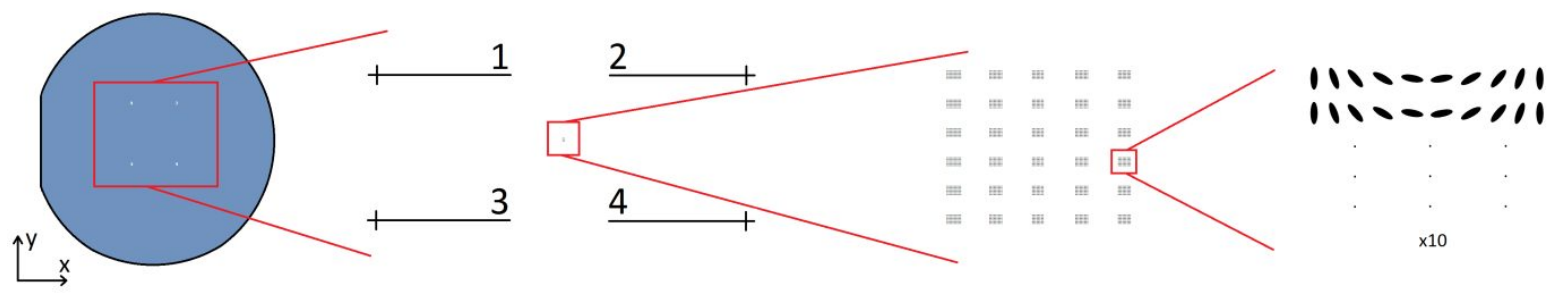

Figure 4 Schematic overview of the programmed defect wafer lay out: stealth fiducial markers enable swift redetection of isolated programmed defects as well as dense fields of programmed defects. From left to right, the image field of view reduces from the $100 \mathrm{~mm}$ scale down to the micron scale. 


\subsection{Programmed defect wafers with etched and metallic model defects}

Defects can occur in a multitude of shapes and forms: particles on top of the substrate, and also holes, pits or scratches in the substrate surface ${ }^{12,13,14}$. The design choices for the programmed defect wafers are somewhat restricted by the involved fabrication processes. Electron beam lithography was used to write rectangular and elliptical defects in CSAR 62 resist $^{15}$, see Figure 5. To emulate particle defects on top of a substrate, metal layer deposition was combined with liftoff. To emulate holes, pitches and scratches, the pattern was etched into the silicon wafer.

Both the etch and the deposition process yield defects with a fixed height. Like the lateral dimension, the height of structures impacts the scatter signal strength. The effect of this parameter was simulated using the FEA scattering model. As a result, an optimal defect height of $20 \mathrm{~nm}$ was found. For lower defect heights, the RN3 signal depends strongly on the exact defect height, which introduces a too high sensitivity to variations in the manufacturing processes. For larger defect heights, the smallest defects get challenging height aspect ratios in manufacturing. Programmed defect arrays with rectangular cuboids and elliptical cylinders that have a large variety in size and aspect ratio were made, see e.g. Figure 5. The lateral dimensions of the defects were chosen to yield a defect volume that is equivalent to that of a cylinder with a diameter of 60,80,100, 200 and 400nm. Both isolated defects as well as dense fields of defects were part of the design. An overview of the programmed defect design parameters can be found in Table 1.

SEM measurements were performed using an FEI NovaNano SEM operated in immersion mode at an electron energy of $10 \mathrm{kV}$ or $15 \mathrm{kV}$. SEM measurements were performed to inspect the defect shape and lateral dimensions. To determine the defect width and length from SEM images, an algorithm is developed to fit the measured SEM waveform with an analytical expression, see the last section in paragraph 3.2.

AFM measurements on the programmed defect wafers were performed with a Bruker Dimension FastScan. The AFM was operated in PeakForce ${ }^{\mathrm{TM}}$ tapping mode and two different AFM tips were used. NanoScope Analysis Tip Qualification software ${ }^{16}$ was used to estimate the tip shape and diameter. This software returns the estimated tip diameter at $5 \mathrm{~nm}$ from the tip end (ETD1) and at 20nm from the tip end (ETD2). Measurements on the etched defects were done with a standard AFM tip with an estimated tip diameter of ETD1 $=80 \mathrm{~nm}$ and ETD2 $=140 \mathrm{~nm}$. Measurements on the metal defects were done with a more accurate high aspect ratio AFM tip ${ }^{17}$ with an estimated tip diameter of ETD1 $=30$ $\mathrm{nm}$ and $\mathrm{ETD} 2=100 \mathrm{~nm}$.

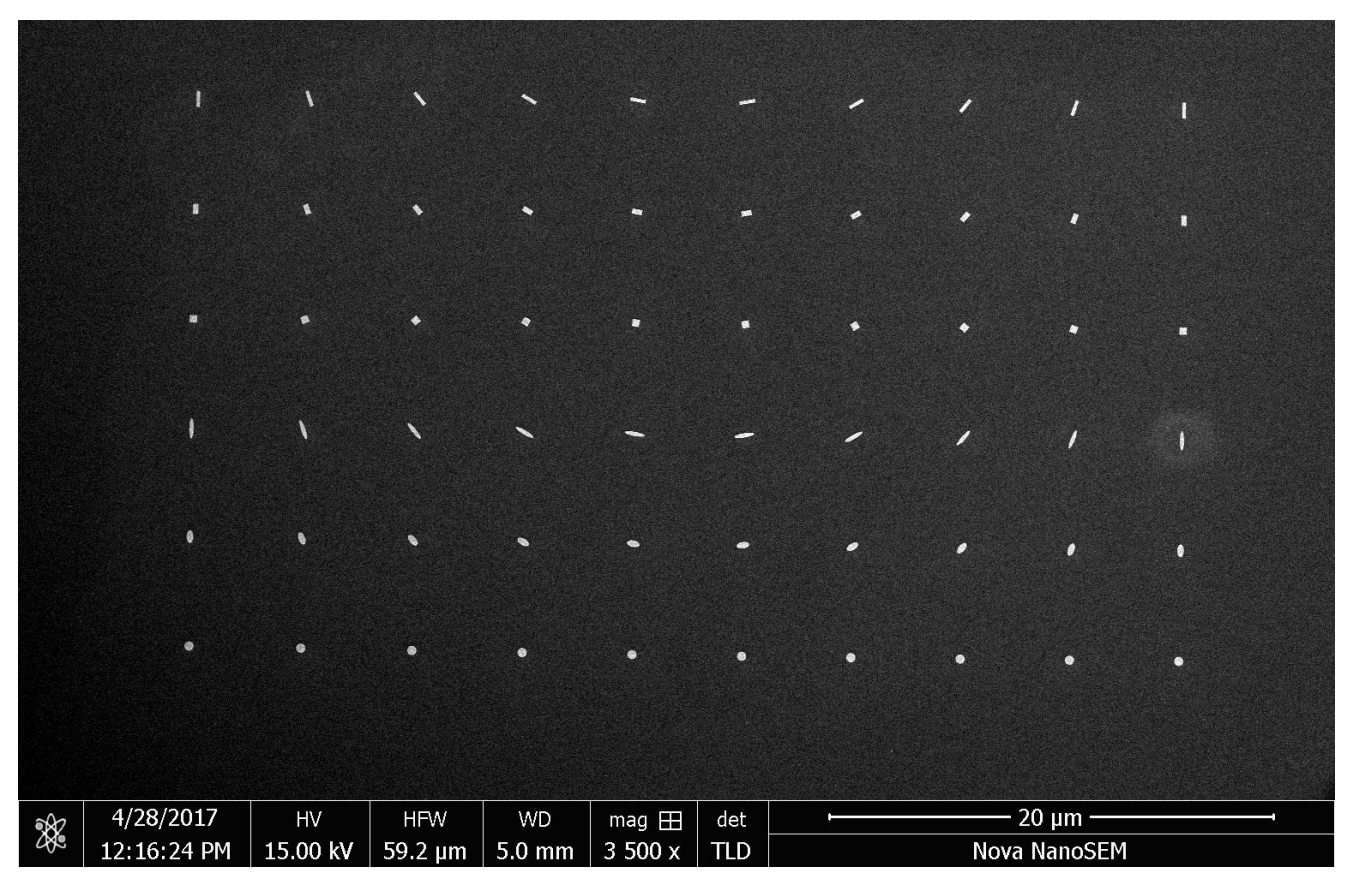

Figure 5 SEM image of a dense field of programmed metal particle defects, containing the same defects at nine different angles. The defects are either elliptical cylinders or rectangular cuboids, and have aspect ratios of 1:1, 1:2 or 1:5. The circle diameter is $\mathrm{CD}=400 \mathrm{~nm}$. 
Table 1 Overview of the variable programmed defect design parameters.

\begin{tabular}{|c|c|c|c|c|}
\hline Defect type & 2D shape & Aspect ratio $(1: \mathrm{AR})$ & Size parameter: D $(\mathrm{nm})$ & Rotation $\left(^{\circ}\right)$ \\
\hline Etched in $\mathrm{Si}$ & Ellipse & $1: 1$ & 60 & $0 / 180$ \\
$\mathrm{Au}$ on $\mathrm{Si}$ & Rectangle & $1: 2$ & 80 & $20 / 200$ \\
& & $1: 5$ & 100 & $40 / 220$ \\
& & & 200 & $60 / 240$ \\
& & & 400 & $80 / 260$ \\
& & & & $100 / 280$ \\
& & & & $120 / 300$ \\
& & & & $140 / 320$ \\
& & & $160 / 340$ \\
\hline
\end{tabular}

Each unique defect type was reproduced 10 times to average out small variations. By measuring all 6000 defects on the two programmed defect wafers, the RN3.1 and 3.9 sensitivity as well as the performance of newly developed ADC algorithms was verified.

\subsection{Algorithms for nuisance filtering and defect shape analysis}

To purify the defect list as obtained after scanning a blank substrate using RN3.1 (see Figure 1), an algorithm was developed to filter out nuisance defect such as e.g. the ghost images of larger objects, or isolated pixels with an unrealistically high grey value. To further enrich the DoI list, a defect shape estimating algorithms have been developed that use RN3.9 data on the asymmetry of the defect scattering. To visualize the asymmetry of the defect scattering, the RN3.9 signals, as recorded for each illumination azimuth, are graphically presented in a polar plot. By example, the three panels in Figure 6 each display a polar plot of the RN3.9 signals as recorded from etched elliptical cylinder programmed defects (see Figure 5) for three different aspect ratios. The raw signals from each arm (red symbols) were normalized (green symbols) using the signals from the circular defects. As an additional check of the calibration method, next to the signals for each illumination arm orientation also the signals from a single illumination arm for similar defects of all orientations (see Figure 5) are plotted (blue symbols). Calibrating the scatter signal from each individual arm proved to be essential to get meaningful results for the defect aspect ratio and size estimation.
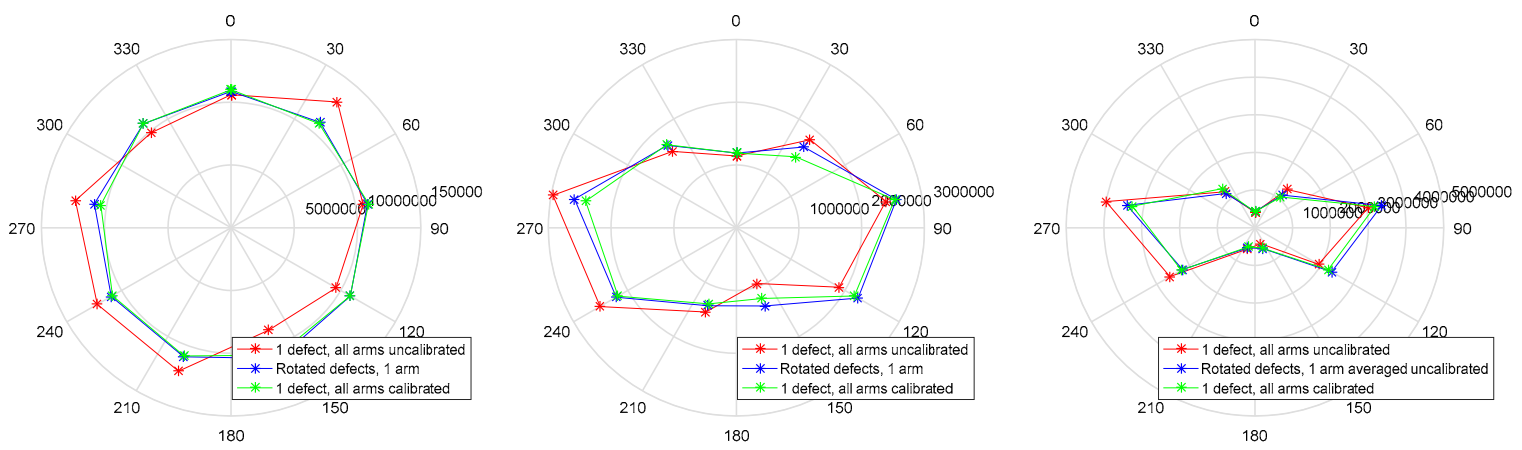

Figure 6 Polar plots of the RN3.9 signal from gold elliptical cylinders with aspect ratios 1:1, 1:2 and 1:5 (left to right panels, respectively). The high similarity between the green (same defect, nine different illumination arms) and blue (same arm, nine rotated defects) contours demonstrates that the signals of all nine arms have been normalized accurately, using the dense defect fields in the programmed defect wafer (see Figure 5). These calibrated signals are used in our algorithms to estimate defect size, skewness and aspect ratio.

\section{RESULTS AND DISCUSSION}

\subsection{Efficient defect redetection in RN3, SEM and AFM}

A defect redetection method that deploys the new stealth fiducial marker system for swift navigation to the DoI sites has been developed and implemented, see Figure 4 and Figure 7. Using laser scribing, an accurate marker system has been applied to clean $100 \mathrm{~mm}$ XXX flat wafers. Figure 7 shows RN3.1, SEM and AFM close-up images of features of the marker system as illustrated in Figure 4. It is noteworthy that indeed the same feature is accurately visible in each 
metrology tool, despite the completely different physics that underlies image formation in each tool. The improved defect redetection enables holistic connectivity of RN3 with high-resolution defect review metrology tools such as AFM and SEM. As a result, both the defect redetection fraction and the defect review speed have increased by more than an order of magnitude for all deployed metrology tools, comprising RN3.9, AFM and SEM ${ }^{6}$. As a result, more than $95 \%$ of the programmed defects could be redetected in SEM and AFM, within the available time per defect.
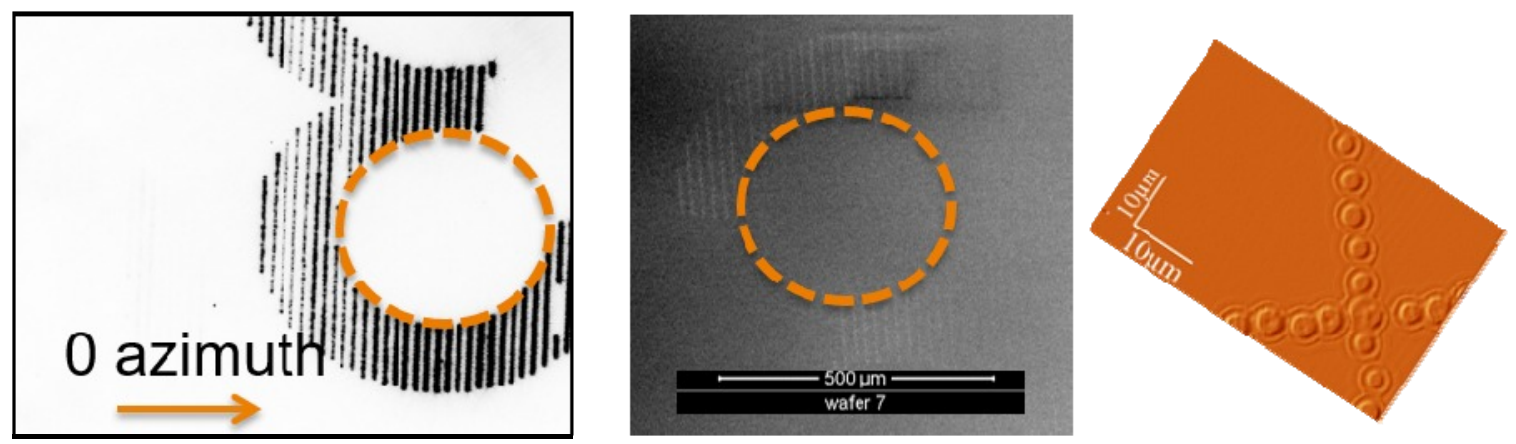

Figure 7 RN3.1 (left panel), SEM (center panel) and AFM (close-up, right panel) images of a laser-scribed stealth fiducial on a clean blank silicon wafer.

\subsection{Validation of RN3.1 and RN3.9 signal strengths by benchmarking experiments with model predictions}

Review of the same defect using different metrology tools, as enabled by the stealth fiducial marker system, can provide useful insights in the accuracy of defect size estimation, as will be illustrated by the examples below. Figure 8 shows the raw signals of RN3.9, SEM and AFM for a typical re-detected programmed defect: an $20 \mathrm{~nm}$ deep etched trench of aspect ratio 1:1, 1:2 and 1:5 in a silicon wafer. The design size of the trenches is $C D=100 \mathrm{~nm}$, where CD is defined as the square root of the length times the width of the rectangular cuboid. The RN3.9 polar plots clearly show that, even when using $532 \mathrm{~nm}$ light, asymmetries in the scattered light due to the defect can be measured for deep sub-wavelength sized defects. Furthermore, using the stealth fiducial marker system, additional information of defect size and shape is available from SEM images, and, to a lesser degree, also from AFM images. For instance, the SEM images show some trench edge roughness, and, as discussed below, provide edge position information that can be used to estimate the lateral trench dimensions. Moreover, the AFM images show that it is difficult to assess the shape of etched defects that have a size that is similar to the AFM probe dimensions. The AFM images show a shadow-like structure in the defect shape resulting from the AFM tip shape.

The COMSOL model was used to predict the optical scatter signal of the same etched defects of Figure 8. Figure 9 shows simulated and experimental polar plots for the RN3.9 signal from these defects. Clearly, there is a good agreement between modelling and experimental results. Noteworthy is that the RN3.1 signal from sub-wavelength sized defects, as can be obtained by summation of the calibrated RN3.9 signals, is significantly stronger for high-aspect ratio defects, although these defects all have the same volume. Hence, by taking defect anisotropy into account, a more accurate value for the defect size in terms of LSE can be reported. This, in turn, is expected to significantly improve the quality of a defect Pareto ${ }^{18}$.

The minor difference between the model prediction and RN3.9 data increases with defect aspect ratio, and provides another example on how the accuracy of a defect size and shape estimate can be improved by analyzing the RN3.9 signals. As outlined in detail below, there is a significant difference between the design size and the actual size of the programmed defects. In short, we attribute the observed differences between calculations and experimental data to differences between the designed and actual defect size, as the COMSOL model used the design CD and aspect ratio, rather than the actual size and shape of the defects. 

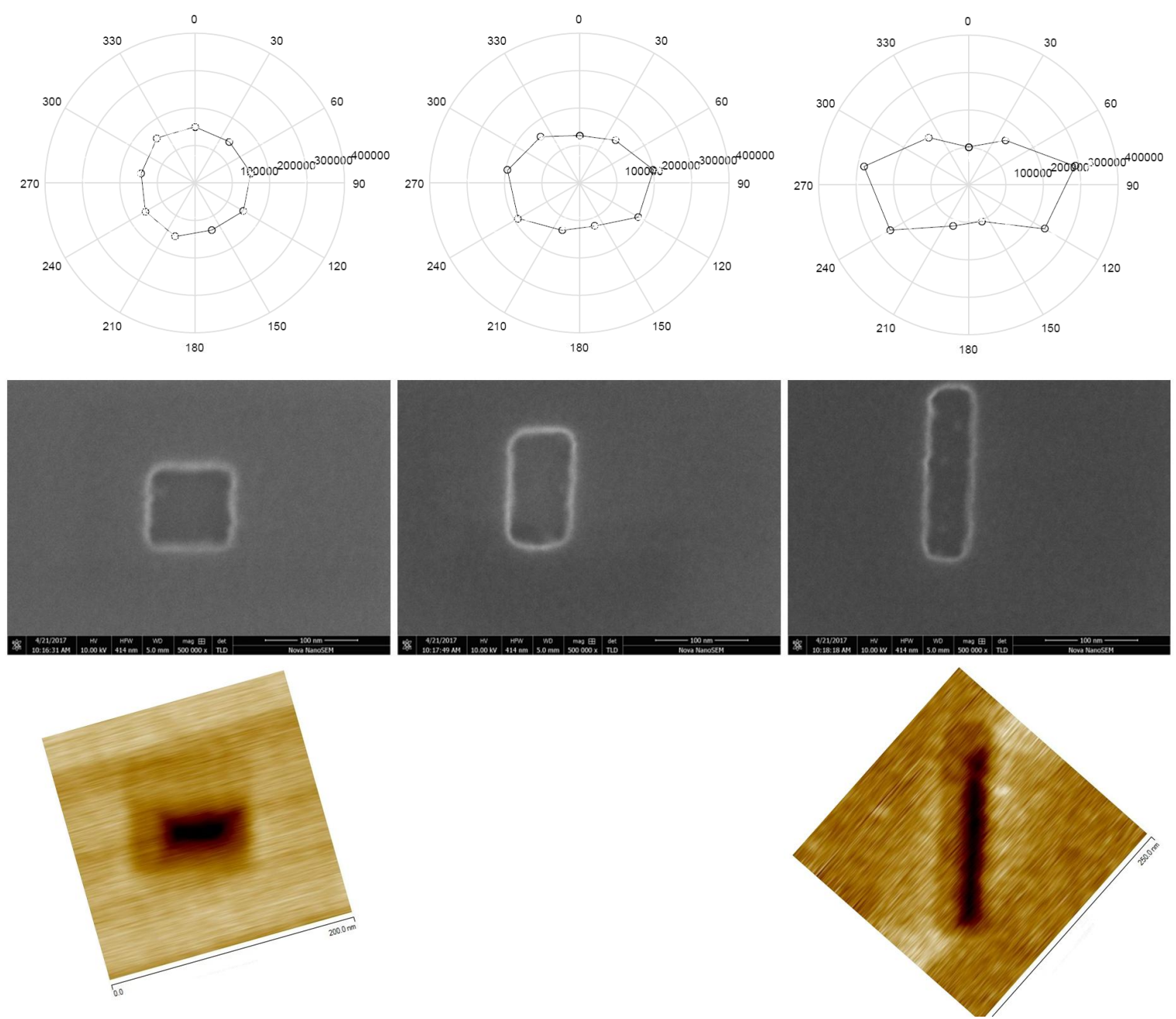

Figure 8: Optical signal strength as measured using RN3.9 (top row, recorded with RN3.9) and SEM (center row) and AFM (bottom row) images, all from the same re-detected programmed defects. The design size of the square defects is $\mathrm{CD}=100$ $\mathrm{nm}$, with aspect ratio $\mathrm{AR}=1: 1$ (left column), 1:2 (center column) and 1:5 (right column).
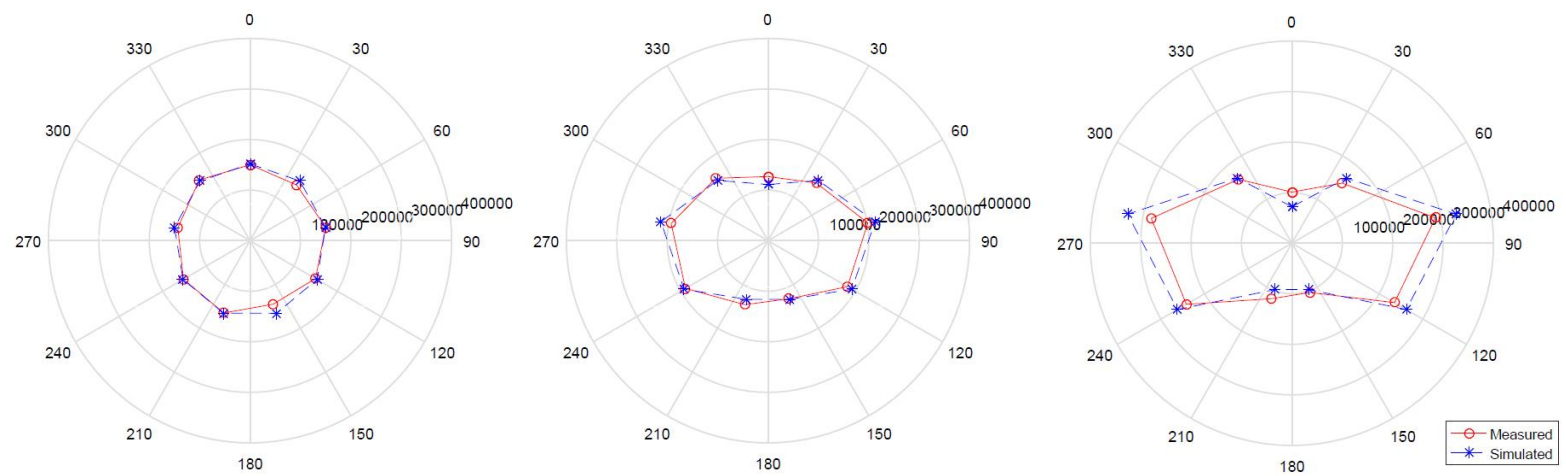

Figure 9 Polar plots with RN3.9 experimental data and simulation results for the optical scattering from the programmed defect of Figure 8. The measured data in the center and right panel show a slightly lower aspect ratio, which is in agreement with the size and AR metrology using SEM images. The qualitative and quantitative agreement between simulation results and experiment demonstrates the validity of the simulation model. 


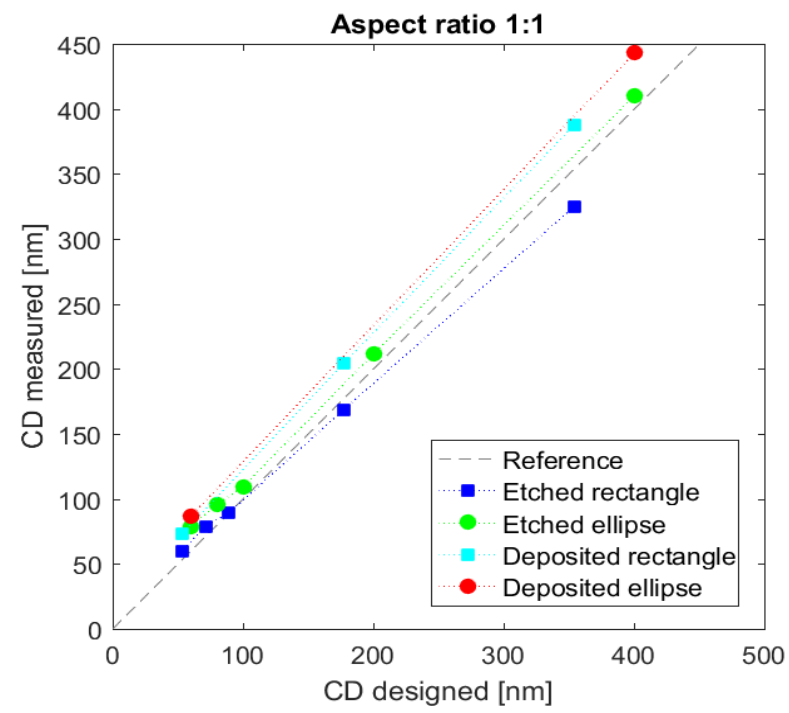

Figure 10 The critical dimensions (CD) of the realized programmed defects as a function of the design CD.

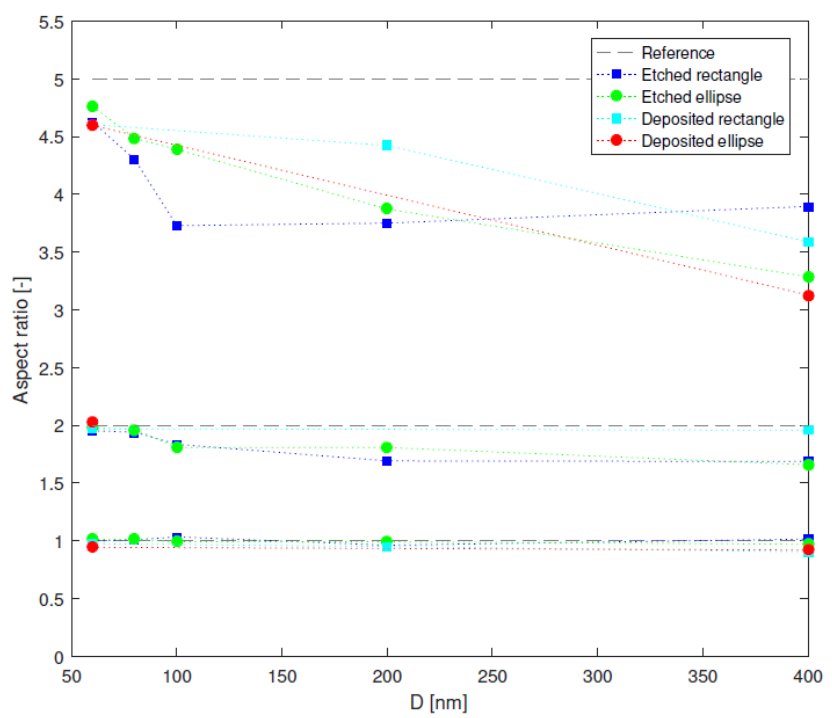

Figure 11 Designed (dashed lines, for reference) and measured (SEM data) aspect ratios of etched and deposited programmed defects. The larger the defect and/or its AR, the larger the difference between the realized and designed AR. The difference is attributed to the proximity effect in electron beam lithography, which mostly broadens narrow features which are most prominent in the highest AR PDs.

It is well-known that in electron beam lithography e.g. proximity effects may change the actual feature size ${ }^{19}$. Moreover, for non-round defects, the feature broadening will impact the actual aspect ratio of the realized defects. Hence, we analyzed the size of realized defects from SEM images, using the Double-Gaussian fit algorithm to SEM waveforms, as adapted by Maas ${ }^{20}$ after Verduin ${ }^{21}$. This algorithm was extended to fit the complete SEM image instead of a single line. The fit result includes the contour of the defect and thus allows for a more accurate estimation of the aspect-ratio. Figure 10 shows how the measured size (i.e. their critical dimension) of the programmed defects as determined from the SEM images deviates from the design CD, especially for defects with higher aspect ratios. Consequently, as shown by Figure 11 , the realized aspect ratio significantly deviates from the design aspect ratio. This effect accounts, at least qualitatively, for the less an-isotropic scatter RN3.9 signal when compared to the simulated scatter profile, as was observed from Figure 9. 


\subsection{RN3.9, SEM and AFM signals from arbitrary shaped defects}

Measurements on field test wafers confirmed that in practice many particles are non-symmetric. For example, Figure 12 shows the RN3.9 polar plot as well as a SEM and AFM image of a typical customer defect on a blank silicon witness wafer. The defects were swiftly re-detected in the SEM and AFM by the usage of the stealth fiducial marker system. The RN3.9 polar plot shows that the defect is non-round, and also a significant non-centricity of the signal. For optical scattering, any particle skewness is expected to cause an angular dependency of the scatter intensity. As a consequence, for a skewed particle, the RN3.9 scatter pattern is not only non-symmetric; also the center-of-gravity of the scatter pattern is shifted from the origin. Hence, we conclude that the defect is non-round and skewed into one direction. These observations are confirmed by the SEM and AFM images. The displacement of the center-of-gravity of the RN3.9 signal from the origin can probably be used as a metric for defect skewness. More RN3.9 and AFM measurements as well as modelling efforts are needed to get conclusive on this subject.

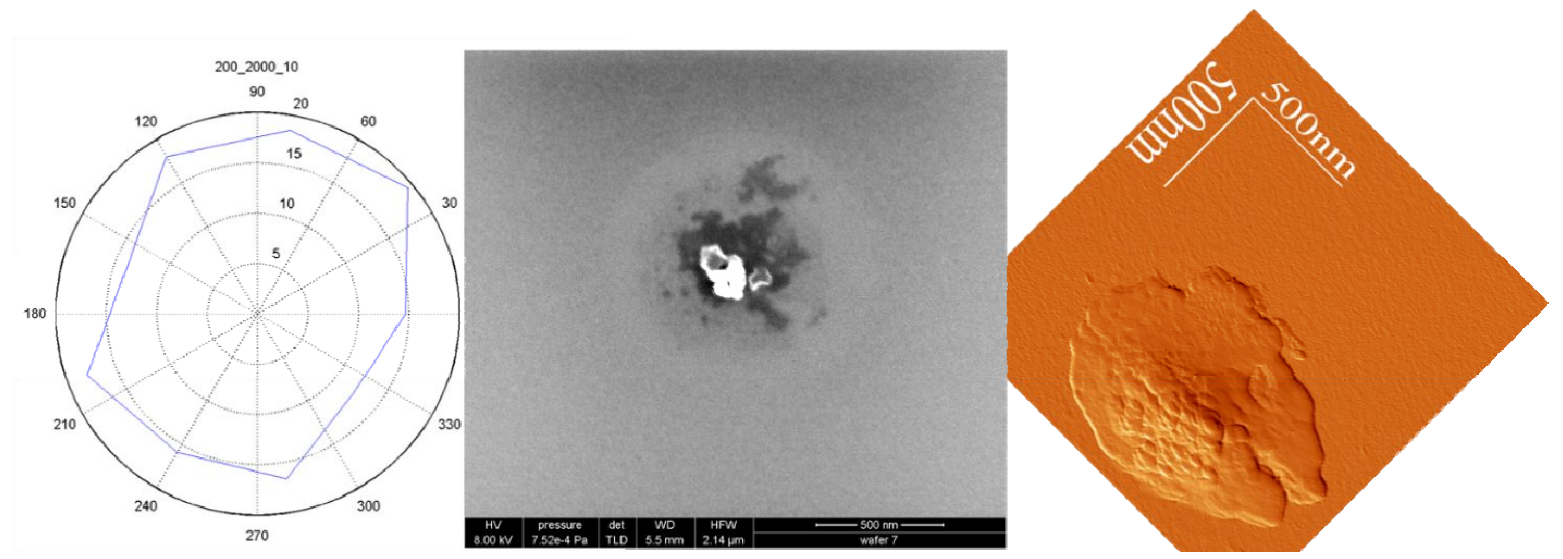

Figure 12 RN3.9 scatter intensity data as well as SEM and AFM images of a "large" particle on a field test wafer. The particle is composed of SiOx (SEM EDX).

\section{CONCLUSIONS}

A process flow for more efficient defect review and classification has been developed. This flow consists of optical inspection to locate the defects, nuisance defect filtering, review by optical metrology to further increase the purity of the defect of interest list and a stealth fiducial marker system with holistic connectivity to high resolution inspection tools like SEM, AFM and HIM. This process flow increases the efficiency of the inspection time on the slower high resolution tools.

We have implemented a new review mode (RN3.9) on our existing particle inspection system (Rapid Nano). This mode gives access to the individual signals from each of the 9 illumination arms of the Rapid Nano. By analyzing this additional data, more properties of the defect can be extracted and better decisions can be made on investing in further review.

Defect review and classification by RN3.9 has been tested on programmed defects of different size, shape, orientation and material. These tests have been performed both with a numerical optical model of the system and with experiments on programmed defect wafers on the actual system. We observed a strong correlation of the programmed defect size and shape metrics as extracted from the RN3.9 signal with SEM and AFM defect metrology data. Furthermore, the RN3.9 signals are in excellent agreement with the predicted scatter intensities by analytical and numerical optical models. Hence it is anticipated that the RN3.9 metrics for defect aspect ratio, skewness and size can be used reliably for advanced defect classification (ADC) of arbitrary-shaped sub-wavelength-sized defects on blank substrates for semiconductor manufacturing applications. 


\section{ACKNOWLEDGEMENTS}

This work was performed in the framework of the E450LMDAP project. TNO gratefully acknowledges funding by the ECSEL Joint Undertaking and the Netherlands Enterprise Agency (RVO). Furthermore, we would like to thank our colleagues Jeroen van den Brink, Christiaan Hollemans, Sjoerd Oostrom and Emile van Veldhoven (TNO) and Kodai Hatakeyama and Marc Zuiddam (Kavli Nanolab, TU Delft) for their skillful assistance in the lab.

\section{REFERENCES}

[1] Boettiger, T., Buck, P., Paninjath, S., Pereira, M., Ronald, R., Rost, D., Samir, B., "Automatic Classification of Blank Substrate Defects", Proc. SPIE 9235 (2014) 92351J.

[2] van der Donck, J., Bussink, P., Fritz, E. and van der Walle, P., "RapidNano: Towards 20nm Particle Detection on EUV Mask Blanks.”, MRS Advances, 1 (2016) 2225-2236. doi: 10.1557/adv.2016.299.

[3] http://www.appliedmaterials.com/files/pdf_documents/SEMVisionG6-technical-briefing.pdf, accessed on June $20^{\text {th }}, 2017$.

[4] https://www.bruker.com/fileadmin/user upload/8-PDFDocs/SurfaceAnalysis/AFM/Webinars/Defect_Classification_by_PeakForce_Tapping_Nanomechanical_Measu rement - FINAL -.pdf, accessed on June $20^{\text {th }}, 2017$.

[5] Alkemade, P.F.A., Koster, E.M., Van Veldhoven, E., and Maas, D.J., "Imaging and nanofabrication with the helium ion microscope of the van Leeuwenhoek laboratory in Delft", Scanning 34 (2012) 90.

[6] Bouwens, M.A.J., Maas, D.J., van der Donck, J.C.J., Alkemade, P.F.A., van der Walle, P., "Enhancing redetection efficacy of defects on blank wafers using stealth fiducial markers.", Microelec. Eng. 153 (2016) 48.

[7] van der Walle, P., Hannemann, S., van Eijk, D., Mulckhuyse, W., van der Donck, J., "Implementation of background scattering variance reduction on the Rapid Nano particle scanner", SPIE Proc. 9050 (2014) 905033.

[8] COMSOL Multiphysics, v5.0, 3D wave optics module.

[9] J. Yang, J.P. Hugonin, and P. Lalanne. "Near-to-far field transformations for radiative and guided waves", ACS photonics, 3(3):395-402, 2016.

[10] https://www.lp2n.institutoptique.fr/content/download/7678/45332/file/NtoFField\%20package\%20V5.zip, accessed on June $20^{\text {th }}, 2017$. Lalane, P., "RETOP: an open Matlab source for near-to-far-field transformation for free and guided waves in optical stacks".

[11] Bobbert, P.A. and Vlieger, J., "Light scattering by a sphere on a substrate.", Physica A 137 (1986) 209-242.

[12] Bohren, C.F., and Huffman, D.R., "Absorption and scattering of light by small particles.", John Wiley \& Sons (2008).

[13] Sugihara, Y., Honda, T., Urano, Y., Watanabe, M., Noguchi, S., and Igarashi, H., "Classification and size estimation of wafer defects by using scattered light distribution", Electr. and Comm. in Japan, 98 (2015) 36-43.

[14] Godwin, M., Balachandran, D., Tamura, T., and Jia, A., "Comparative defect classifications and analysis of Lasertec's M1350 and M7360", Proc. SPIE 9050 (2014) 90502Z.

[15] Allresist. Positive e-beam resists ar-p 6200 (csar 62). Datasheet (2017).

[16] Bruker. Nanoscope analysis 1.50 user manual, (accessed: 19.05.2017).

[17] NanoAndMore GMBH. Afm probes, isc-125c40-r. URL: https://www.nanoandmore.com/ AFM-Probe-ISC125C40-R.html, (accessed: 19.05.2017).

[18] http://whatis.techtarget.com/definition/Pareto-chart-Pareto-distribution-diagram, assessed on June 22, 2017.

[19] Mohammad, M.A., Muhammad, M., Dew, S.K., and Stepanova M., "Fundamentals of Electron Beam Exposure and Development", in "Nanofabrication", Stepanova M., and Dew, S. (eds.), DOI 10.1007/978-3-7091-04248_2, Springer-Verlag/Wien (2012).

[20] Maas, D.J., Fliervoet, T., Herfst, R., van Veldhoven, E., Meessen, J., Vaenkatesan, V. and Sadeghian H., "Sub$50 \mathrm{~nm}$ metrology on extreme ultra violet chemically amplified resist - A systematic assessment.", Rev. Sci. Instr. 86 (2015) 103702; doi: 10.1063/1.4932038.

[21] Verduin, T., Kruit, P., and Hagen, C.W., "Determination of line edge roughness in low dose top-down scanning electron microscopy images”, Proc. SPIE 9050, 90500L (2014). 